\title{
BIOINSPIRED COMPUTATIONAL DESIGN: A CASE STUDY ON A 3D-PRINTED LAMP BASED ON THE PHYSALIS ALKEKENGI
}

\author{
Graziosi, Serena; \\ Scurati, Giulia Wally; \\ Parmose, Roberta; \\ Lecchi, Alberto; \\ Bordegoni, Monica; \\ Ferrise, Francesco \\ Politecnico di Milano
}

\begin{abstract}
Nature has always been a source of inspiration for designers and engineers, through the imitation of biological patterns and structures. This emulating and creative process is nowadays supported by technologies and tools as additive manufacturing and computational design. This paper describes the design and prototyping of a lamp inspired by a plant called Physalis Alkekengi, known as Chinese Lantern. We present the development of an algorithm, based on a computational model from literature, to realize the $2 \mathrm{D}$ pattern and leaves. They were then $3 \mathrm{D}$ printed to create the structure of the lamp and obtain an aesthetical and symbolic shading effect.
\end{abstract}

Keywords: Computational design methods, Design for Additive Manufacturing (DfAM), Bio-inspired design / biomimetics

\section{Contact:}

Graziosi, Serena

Politecnico di Milano

Department of Mechanical Engineering

Italy

serena.graziosi@polimi.it

Cite this article: Graziosi, S., Scurati, G. W., Parmose, R., Lecchi, A., Bordegoni, M., Ferrise, F. (2021) 'Bioinspired 


\section{INTRODUCTION}

An underlying order permeates the natural world. Philip Ball examined these organisational aspects and analogies between them, considering patterns and shapes in organisms (Ball, 2009b), the presence of branches in biological and geological systems (Ball, 2009a), and mechanisms through which these repeating features are generated (Ball, 2011). Clearly, nature has always being a source of inspiration for humans engaging in any creative discipline. Artists and artisans, architects and designers, and also scientists and engineers, have reproduced, studied and elaborated, a variety of natural shapes and structures. Organic forms and biological geometric patterns are used for their functional properties, aesthetic qualities, and symbolic meaning. This broad spectrum of possibilities was described by Volstad and Boks (2012), that created different categories based on the inspiring concepts (material, mechanics/dynamics, structure and form) and the products of the activity and field of application (material science, engineering/locomotion, structural engineering/architecture and art/architecture).

All these trends can be collected under the umbrella of biomimicry, defined as innovation inspired by nature (Benyus, 1997). This concept has triggered product innovation in many ways. It allows to obtain specific mechanical properties and performances, through the emulation of material structures (Fischer et al., 2010), but also systems and mechanisms (Sihite et al., 2020). It can be thus a means to explore both new material designs (Ren et al., 2019) and various aesthetic and functional features, as in the case of furniture (Tavsan and Sonmez, 2015) and architectural design (Oxman, 2010) or innovative wearable solutions (Babaee et al., 2020). In general, it can support design thinking processes, with proposals of design toolkits for professionals and education (Volstad and Boks, 2012; Stevens et al., 2020). Finally, it is a driver for sustainable innovation: low energy survival systems are common in the biology world. This principle can be applied in the design of more efficient products (Kennedy and Marting, 2016) and buildings (El-Zeiny, 2012) to reduce the consumption of resources. Several sustainable applications can also be found in the textile industry: biomimicking is an instrument to maintain or achieve new functional properties while reducing the use of toxic chemicals (Weerasinghe et al., 2019).

In technical fields, the evolution of biomimicry tendencies cannot be considered an isolated phenomenon. This nature-inspired innovation has been continuously enabled by other synergetic innovations, with the introduction of new materials, technologies and tools from the ideation to the implementation of new designs. The study of natural elements, at a micro and macro scales, is facilitated by the development of techniques to observe the morphology and structure of animals and plants, leading to reverse engineering processes (du Plessis and Broeckhoven, 2019). Advanced fabrication technologies as Additive Manufacturing (AM) allowed producing a wide variety of organic shapes that are not achievable with other production methods. Hence, the rapid evolution of AM technologies and materials is continuously stimulating nature-inspired Design for Additive Manufacturing (DfAM) studies, exploiting different biological creatures (Yang et al., 2018; Wang et al., 2020). For example, in (Fernandes et al., 2020), the skeleton of deep-sea sponges has inspired the design of buckling-optimised 3D-printed lattices that could, in turn, also improve the design of large-scale structures, such as bridges. The combination of biomimicry researches and AM technologies has inspired several breakthrough solutions also in the field of soft robotics (Schaffner et al., 2018). Moreover, the design process of these applications takes advantage from the use of computational tools and approaches. They allow to analyse, model, replicate and elaborate complex forms and structures (du Plessis et al., 2019). An example is provided by Salcedo et al. (2019), that describe the computational analysis of insects' wings, considering different morphological features (contour, curvature, venation segments and shapes created by them). Besides, several computational models of plant architecture (Prusinkiewicz and Hanan, 1989) are already available in the literature.

The use of biomimicry can follow top-down or bottom-up approaches. The former starts with the definition of a design problem, and it continues with the search for biological analogies to reach a solution. At the same time, the latter begins with biological research, identifying and transforming natural principles in a technical solution for an existing problem (Aziz and El sherif, 2016). In this work, we present a case study of the design and prototyping of a lamp based on a plant named Physalis Alkekengi, adopting a bottom-up approach. This plant is commonly known as Chinese lantern because of its colourful sheen, its airy shape, and the thinness of its leaves quite alike tissue paper. This peculiar meaning and resemblance inspired the proposed solution. This paper is structured in the following way: in Section 2 we describe the methodology we used and the morphogenesis of the leaf venation pattern, particularly 
diving into the algorithmic method proposed by Runions et al. (2005) tailoring the procedure to our case. Finally, in Section 3, we discuss the results obtained, while Section 4 is dedicated to conclusions and possible future developments of our work.

\section{MATERIALS AND METHODS}

\subsection{Methodology}

In this work, we used a methodology based on a bottom-up approach (Aziz and El sherif, 2016). The first step is to identify an inspiration in the natural world, that can be functional, aesthetic, or symbolic. In the second step, a design context should be identified (e.g., main functionalities, aesthetical properties, and target users). Then, as a third step, a computational model of the desired natural features is created. This model can also be generated starting from literature studies of the natural organism or phenomena. Since the model can be quite complex, it is essential to define the context in phase two clearly: in this way, the model can be developed or adapted depending on the specific application and design targets. Finally, in the fourth step, this model is implemented into a design solution. The methodology is illustrated in Figure 1. Hereafter, we explain how this methodology was applied in our case study.

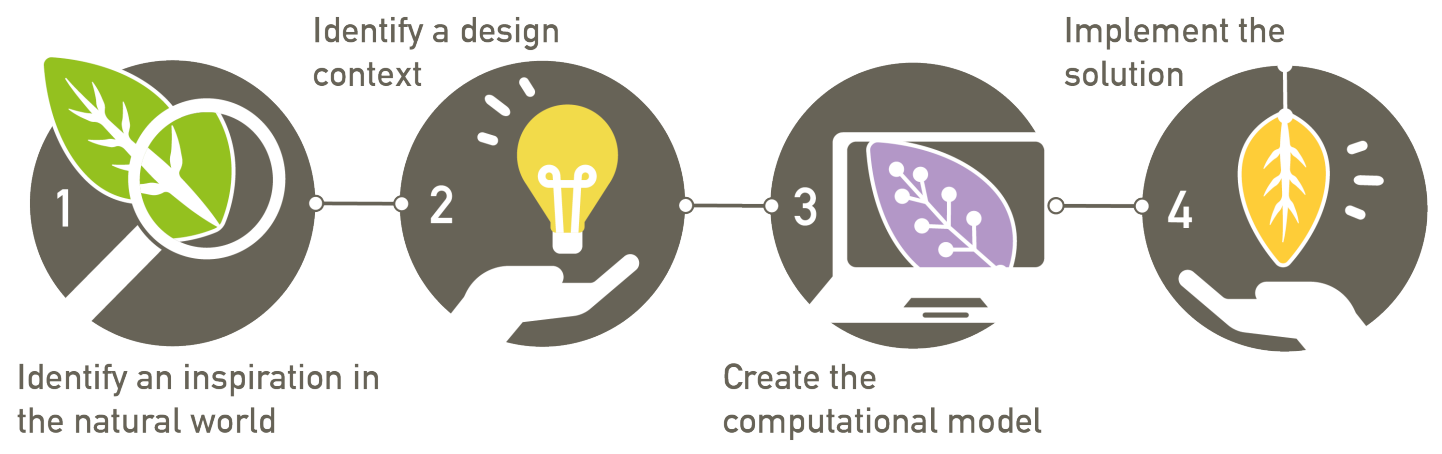

Figure 1. Methodology: steps to follow.

\subsection{The case study: Physalis Alkekengi}

The first step of the methodology led to the identification of the Physalis Alkekengi (Figure 2), that is a plant having an orange berry encapsulated in a peculiar calyx, defined as inflated calyx syndrome ( $\mathrm{Li}$ et al., 2019) and consisting of five leaves. They are green initially, then gradually turn off a fiery red and, when fully ripe in August/September, wither drawing an intricate web of veins presenting chinks that let glimpse the orange berry. The scientific reason behind this fruit enveloping calyx was investigated by Li et al. (2019). They found that the lantern structure of Physalis has different functions and properties, including photosynthetic capabilities (serving as the energy source for fruit development), providing a micro-climate for the growth and maturation of the berry, and facilitating the dispersal of fruits and seeds by water and wind. As mentioned before, this plant is also known as the Chinese lantern (Li et al., 2019) due to its shape, color, and structure. Its symbolic meaning, and aesthetical features, defined the second step of the methodology (Figure 1). The design context was identified, choosing to design a lamp. Specifically, the appearance of the Alkekengi suggested the design of ambient decorative lights rather than a functional one (e.g., a desk lamp). Indeed, the transparencies of the dry calyx of the Alkekengi thickly draped with veins inspired the design of a lamp that could replicate the interplay of lights and shades of its leaves and where the berry is a led light bulb. To this end, we start exploring how the biological model, describing the creation and evolution of the venation pattern of a leaf blade, had been already formalised in the literature (third step). Based on this exploration, we started from the computational model described in (Runions et al., 2005) to develop a Grasshopper ${ }^{\circledR}$ (www.grasshopper3d.com) algorithm within the software Rhino (www.rhino3d.com) to generate the digital and then the physical model of the lamp (Figure 1, fourth step). The computational model of Runions et al. (2005) was adapted to our specific design context. Finally, the Ultimaker 3 (www.ultimkaer.com) was used to print the 3D model, that was then assembled to complete the lamp prototype. 


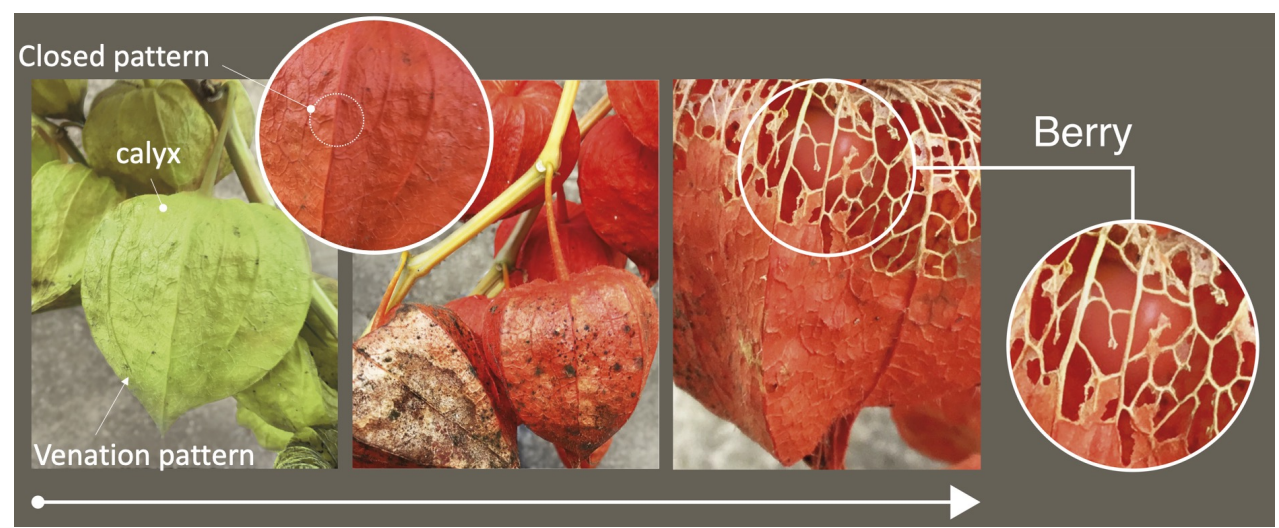

Figure 2. The evolution of the Physalis Alkekengi calyx.

\subsection{The biological computational model}

The third step (Figure 1) started with a literature review of existing modelling techniques. The morphogenesis of plants has been described in years towards multiple computational techniques (Prusinkiewicz and Rolland-Lagan, 2006). One of these techniques is represented by the use of mechanistic simulation models (Prusinkiewicz and Rolland-Lagan, 2006), aiming to formalise the mechanism, and the interaction among its main elements, that leads to the generation of the form. Some of the various mechanistic models available implement the auxin-driven morphogenesis process in which the development of the plant is based on the active transport of auxins (Prusinkiewicz and Rolland-Lagan, 2006; Zhao, 2010). Auxins are the hormones that guide the development of vein nodes (Runions et al., 2005).

Concerning the modelling strategy, the Lindenmayer systems (L-system) mathematical formalism, introduced in 1968 (Prusinkiewicz and Lindenmayer, 2012), has been amply used and extended in years to develop mechanistic models (Prusinkiewicz and Rolland-Lagan, 2006) in the field of plant biology. One of the first attempts to formulate a mechanistic model in geometrical terms is the work of Runions et al. (2005) (Prusinkiewicz and Rolland-Lagan, 2006). This interesting attempt has stimulated the possibility to exploit these models for the generation of innovative shapes, inspired by the morphogenesis of plants, such as the 3D-printed Hyphae Lamps designed by the Nervous System (2011) studio, or new building envelope systems (Gokmen, 2013). In our study, we also exploited the computational model described in (Runions et al., 2005) to generate the venation pattern of the Alkekengi lamp. This model implements the auxin-driven morphogenesis within a feedback process which also includes the leaf growth (Runions et al., 2005). The hormone auxin (i.e., the source) drives the development of the veins which determine the placement of new sources, while the leaf blade continues to grow.

We decided to implement some simplifications to the model of Runions et al. (2005). Their algorithm generates 2D shapes, while we intended to obtain the 3D model of the leaf. For this reason, to optimise computational efforts, in our algorithm, the leaf does not grow together with the venation pattern. It is kept fixed across all the steps of the algorithms, and it is modelled as a boundary profile. The second simplification is related to the placement of the auxin sources. They are defined at the beginning and modelled as discrete points located randomly along the leaf blade. On the contrary, in (Runions et al., 2005), the placement of the auxin sources is dynamic, because it depends on the kinetics of the leaf growth. However, still the auxins biosynthesis process is currently a research topic in botany (Zhao, 2010). The third simplification is related to the structure of the venation pattern. Indeed, we did not enforce the closeness of the leaf pattern (Figure 2), i.e., venation can be modelled as open polylines running through the leaf blade (closed venation is modelled as closed polylines). In (Runions et al., 2005) closed patterns are also considered, but an ad-hoc algorithmic approximation is implemented.

The algorithm starts with the definition of the 2D boundary of the leaf and 1 node placed at the bottom of the leaf (Figure 3a). The node represents the origin of the veins. Then, $n$ auxins are randomly generated on the leaf blade (they are 3 in Figure 3b). Auxins can be active, i.e., they work as points of attraction for the growth of the vein, or inactive, if they do not affect the outline of the venation. In the beginning, all the auxins are active. Simultaneously, the initial node starts to generate the veins whose length $t$ is defined apriori (Figure 3c). At the extremity of the vein, a new node will appear (Figure 3c). An auxin becomes inactive if a vein reaches it. This phenomenon occurs if the distance between the auxin and the node is smaller than a threshold value called kill distance. Each auxin is associated with the vein 

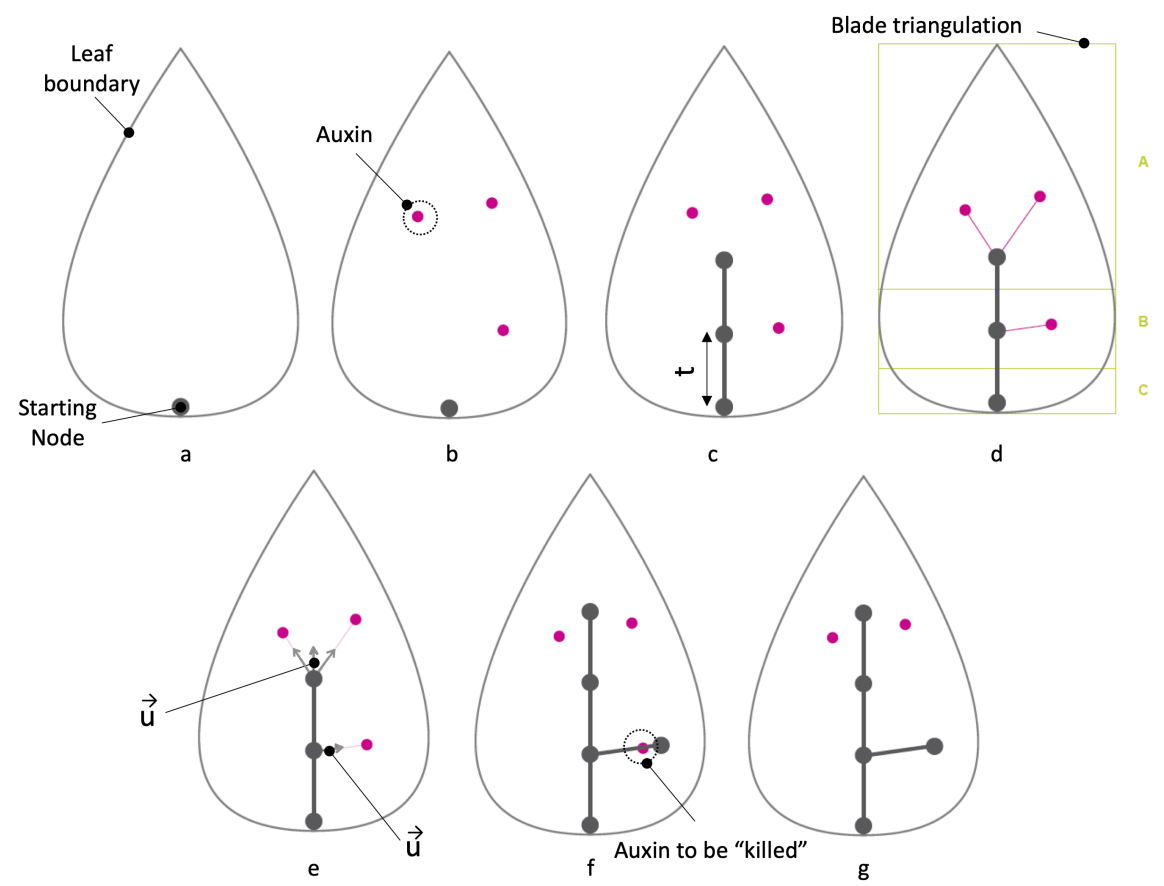

Figure 3. Schematic of the computational model used to generate the venation pattern. Image inspired by (Runions et al., 2005)

node that is closest to it through a Voronoi triangulation of the blade (Figure 3d). For each vein node, a set of active auxins is defined as those that lie in the Voronoi cell containing the node. A vector $\vec{u}$ is computed by averaging all the normalised vectors linking the node with the auxins clustered in the same Voronoi cell (Figure 3e). The vector $\vec{u}$ is used to generate the new vein (Figure $3 \mathrm{f}$ ). The rationale of this procedure is to let the veins growing according to the attraction exerted by the auxins on the nodes. The use of Voronoi cells guarantees that only the auxins closest to a vein node attract it. The last step deactivates those auxins that have been reached by a vein (Figure $3 \mathrm{~g}$ ). This is done by computing the distance between auxins and vein nodes and checking if their distance is below the kill distance. Then, the process continues as a loop until all the auxins are deactivated or, a maximum number of iterations is reached. The parameter $t$ and the number of iterations significantly affect the overall outline of the venation. We have tuned these parameters until a satisfactory result was achieved. Hence, the inputs of the algorithm are: the blade of the leaf represented as a 2D boundary region; the kill distance; the vein length $t$, which is also the step size; the maximum number of iterations $\left(k_{\max }\right)$; the number of auxins.

\subsection{Development of the Grasshopper algorithm}

The third step (Figure 1) continued with the development of the computational model to create the leaf venation pattern within the Grasshopper environment. First the 2D boundary of the leaf is created (Step 1, Figure 4) through 6 points defined through X-Y coordinates. One of these points, the one at the base of the leaf, is selected as the Starting NODE (Step 1, Figure 4). Then, the surface is populated using the Grasshopper Populate Geometry command, renamed here as "Auxins" which randomly populates the geometry "G" (i.e., the leaf surface). In this case, 130 auxins (Step 2, Figure 4) are added. The value of the random index (i.e., 78) specifies the selected random "position" of the auxins. We also defined some extra points on the leaf curves to force the veins to stop in the boundary of the leaf.

The first vein is created (Step 3, Figure 4) using as inputs the Starting NODE and the auxins and by implementing the following strategy. First, the vectors connecting all the auxins with the starting node are generated. Then, an average direction is calculated from these vectors. Hence, the new vein is created using this average direction while its length is determined by the selected step size $t$ which, in this case, is $2 \mathrm{~mm}$. The iterative cycle (Figure 5) is implemented through the Grasshopper Hoopsnake plug-in (www.food4rhino.com/app/hoopsnake). The average direction of the vectors between a node and its associated auxins is found for each new node (as done for the generation of the first vein). This association is performed through the Voronoi subdivision. When the new vein is created, a new line is added and consequently a new node (i.e. a point). The HoopSnake command takes as input 
1_Definition of the leaf boundary
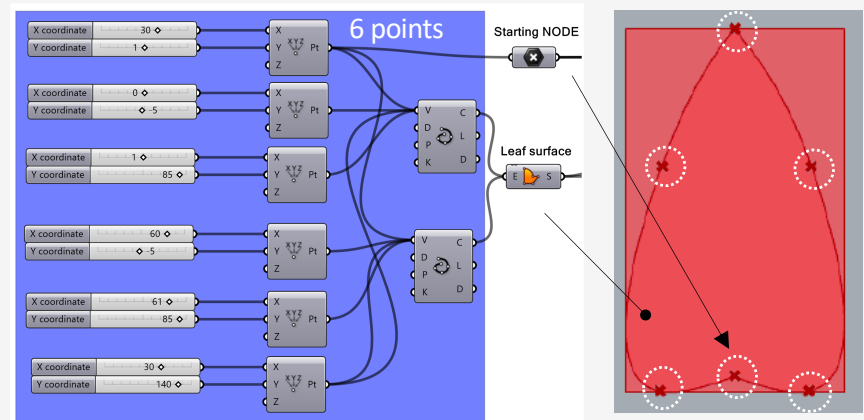

2_Polulation of the blade with auxins

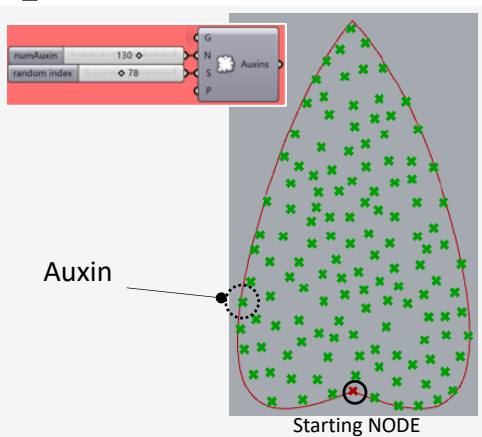

3_Creation of the first vein

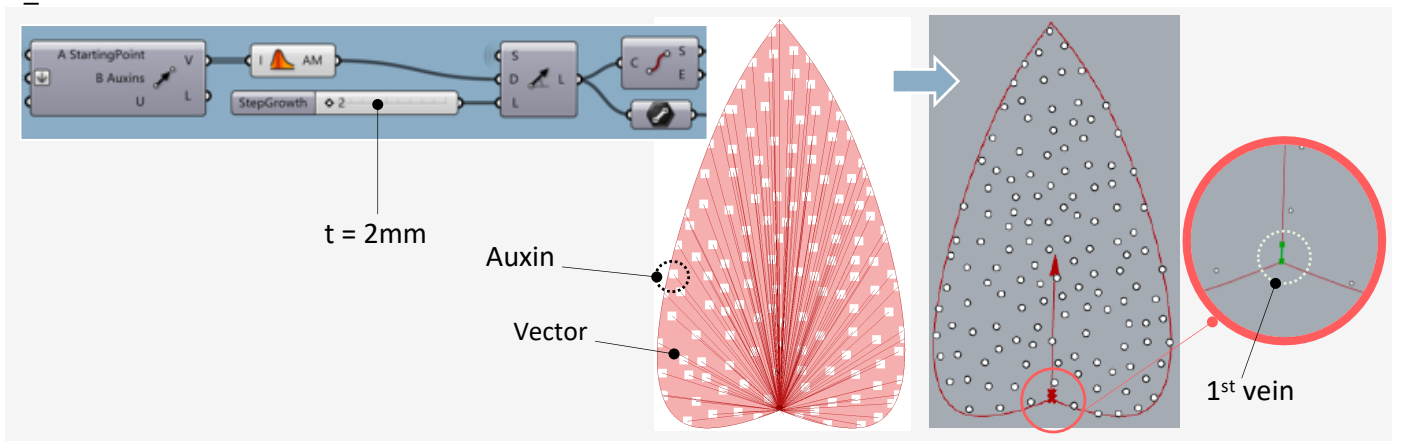

Figure 4. The first three steps of the Grasshopper algorithm.
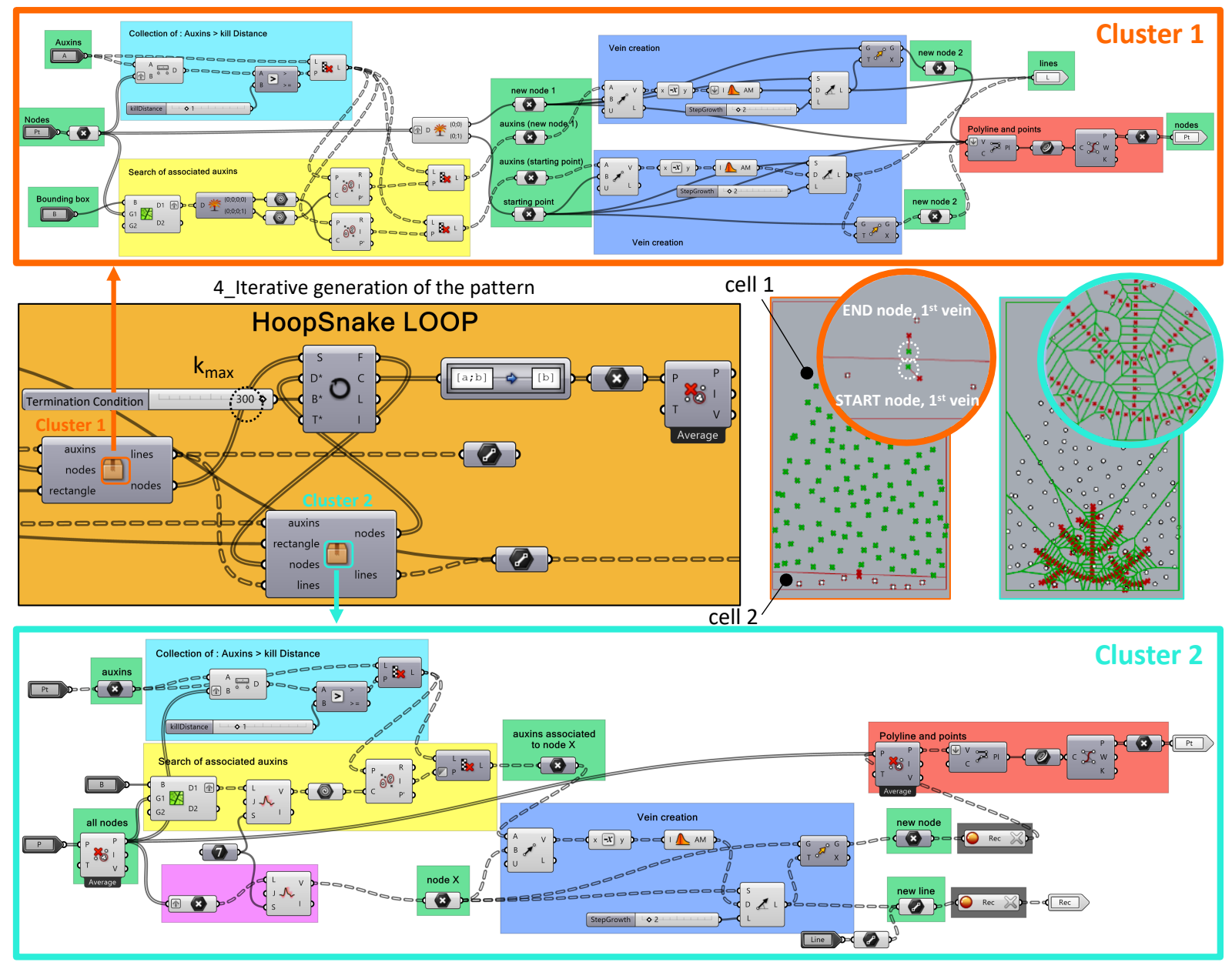

Figure 5. The fourth step of the Grasshopper algorithm: how the Hoopsnake loop works. 
the nodes which are the outputs of the Clusters 1 and 2, i.e., $S$ and $D$, respectively (Step 4, Figure 5). The term Cluster is used to indicate a collection of sub-functions. The sum of the inputs (S+D) determines the Feedback output $F$ which is the input of Cluster 2 and represents the nodes around which the Voronoi subdivision of the bounding box that circumscribes the boundary of the leaf shall be created. The maximum number of feedback loops is set to $300\left(k_{\max }\right.$, Figure 5$)$. The overall output of the Hoopsnake cycle is the Cumulative Feedback (C) that contains all the nodes belonging to the veins of the 2D pattern. The graphical outputs are the lines (i.e. the veins) which are collected and accumulated within the Cluster 2.

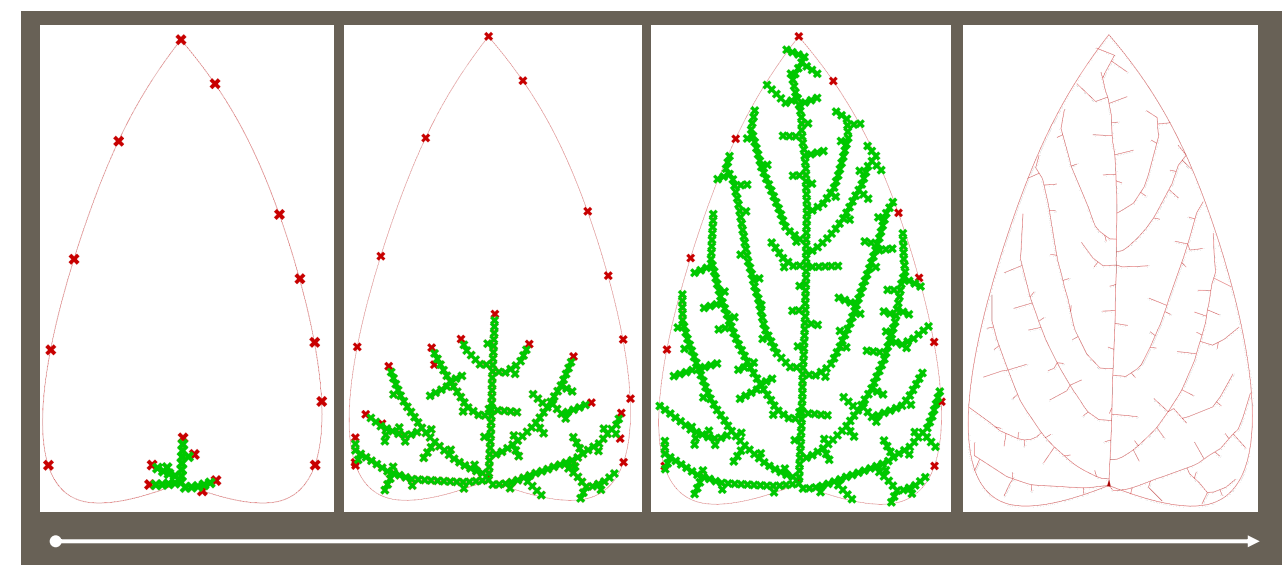

Figure 6. Overview of the venation pattern evolution.

In the Cluster 1 (Figure 5, top), first, the distances among the auxins and the nodes are calculated: the auxins having a distance, from the existing nodes, which is smaller than the defined killDistance (i.e., $1 \mathrm{~mm}$ ) are eliminated. Afterwards, the remaining auxins are associated with the corresponding nodes (i.e. the starting and ending point of the first vein). The assignment is done by dividing the bounding box with the Voronoi cells (two in Figure 5) created for each node. After having found the auxins associated with each node, the process continues with the creation of veins for both nodes (as done for the first vein, Figure 4). From these veins, new nodes are obtained (only if auxins are present in the cell induced by the node; otherwise, no vein is formed). These nodes are the inputs for Cluster 2 and in particular of its Voronoi function. In the Cluster 2 (Figure 5, bottom) these new nodes undergo the same steps of Cluster 1 (elimination of the auxins whose distance from the reference nodes is smaller than the killDistance and subdivision of the bounding box into Voronoi cells). One node per time is selected and analysed, and all the veins (i.e., lines) created for each iteration are recorded. At the end of the HoopSnake cycle, the 2D pattern of the first leaf is created (Figure 6). As already underlined, we generated open patterns. To create closed patterns, we would have modified the algorithm, i.e., the nodes can also be attracted by auxins belonging to adjacent nodes when they are relatively close. In this way, the new veins generated by the nodes would meet at the same point, creating a closed pattern. This path has not been considered in our study because of the excessive computational resources required for elaborating this condition.

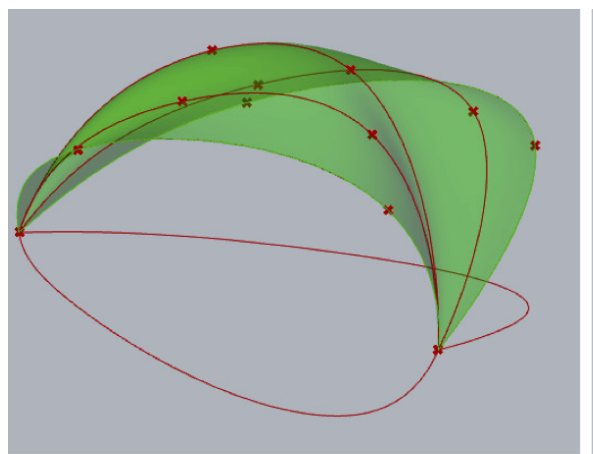

a

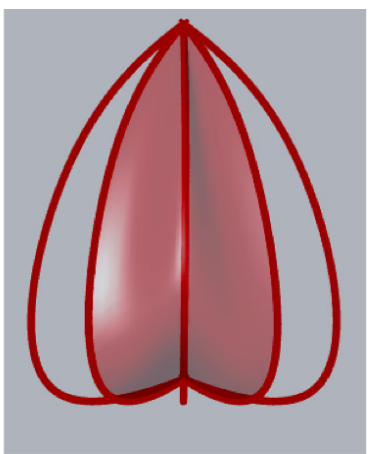

b

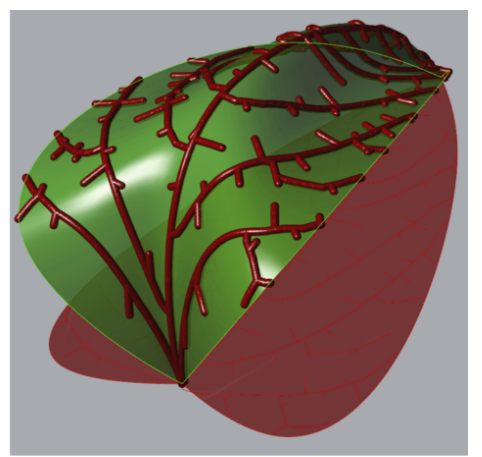

C

Figure 7. The generation of the 3D model: a) the 3D surface of the leaf; b) extrusion of the leaves boundaries; c) extrusion and projections of the veins. 
We then started tailoring the computational model to our design target, i.e., the generation of the 3D model of the lamp (fourth step, Figure 1). All the steps represented in Figures 4 and 5 are repeated 5 times because the Alkegengi has 5 leaves. Each leave differs from the others due to the different random positions of the auxins. This change was implemented on purpose, to create leaves with different patterns. The 2D geometry of each leaf is then transformed into a 3D surface by using a Loft surface command (Figures 7a). The extrusion of the 3D boundary leaf (Figures 7b) is performed using the Grasshopper Cocoon add-on (https://grasshopperdocs.com/addons/cocoon). The 2D pattern is then projected on the 3D surface and veins are extruded (Figures 7c). All 5 patterns are projected on the same 3D surface (only 1 surface was generated) and then rotated of different angles to cover the whole Alkekengi calyx (Figures 8). Two versions of the lamp can be generated, i.e. with or without the leaf surface; they represent two different phases of the calyx development (Figure 2).

\section{RESULTS AND DISCUSSION}

Since we decided to print the lamp using a Fused Filament Fabrication (FFF) 3D printer, the closed version of our lantern was selected (Figure 8a, left). To print the open version (Figure 8a, right), other AM technologies, such as the Selective Laser Sintering (SLS) or the Stereolithography (SLA), would be more appropriate. A small version of the lamp was 3D-printed, without the need of supports and using the transparent Ultimaker PC (polycarbonate) filament, which is a polymeric material amply used for manufacturing lamps. Figure $8 \mathrm{~b}$ shows the printed version of the lamp $(65 \mathrm{~mm}$, height), while Figure 9 shows the prototype within a virtual context. The resulting product is a lamp able to create a pattern of light and shadow through the web of veins that characterises the Alkekengi. The walls of the room are transformed into delicate petals creating the illusion of being safe inside, like its fruit (Figure 2).
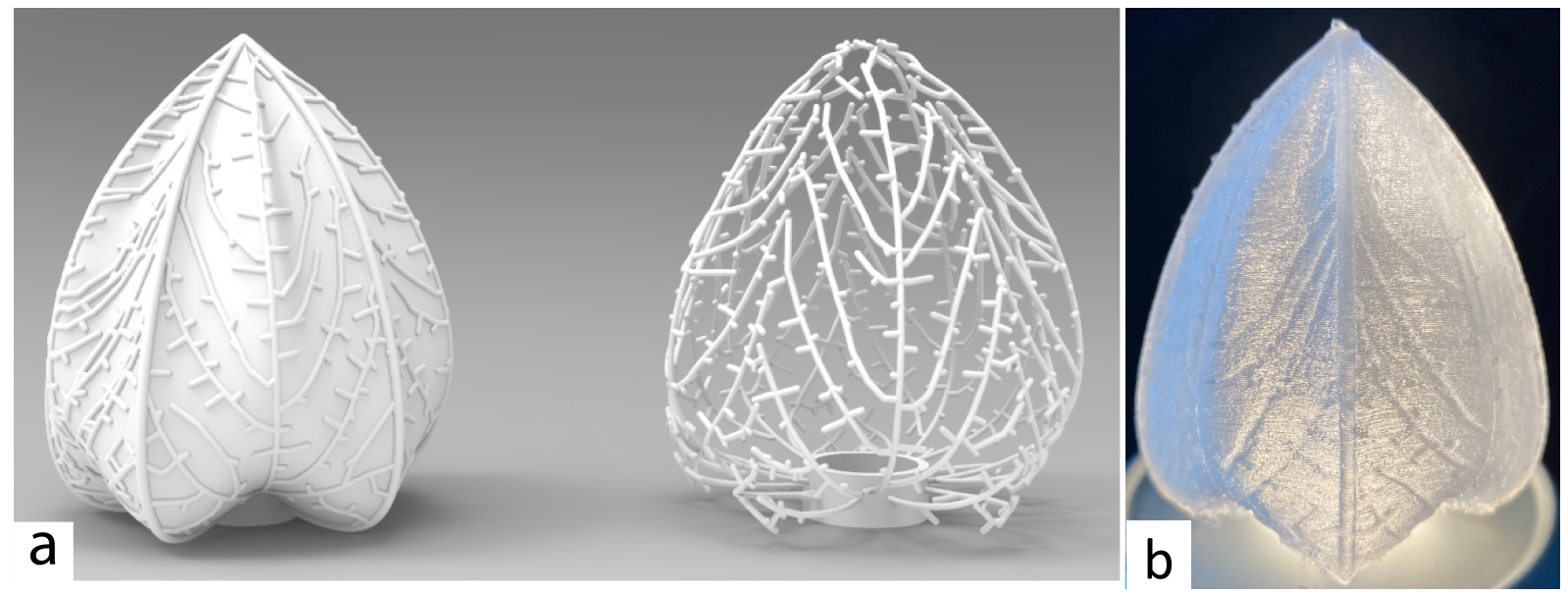

Figure 8. a): Two versions of the Alkekengi lamp 3D model. b): The printed version.

This case study has stimulated several reflections. First, the developed computational model allows for creating unique products. Different venation patterns can be quickly generated, even for each leaf, by changing the number of auxins and the index related to its random distribution. Also, other parameters could be easily modified, such as the vein length $t$, to develop different patterns. That is an appealing design opportunity which is also supported by the design freedom allowed by AM technologies. Second, the developed algorithm could be extended to include manufacturing constraints to keep under control the manufacturability of the multiple variants of the lamp that the algorithm can generate. Third, although some simplifications have been implemented to develop the proof of the concept of the lamp, this approach has demonstrated the possibility to use the models describing biological phenomena as generators of new ideas of products. However, to reach this target, the proper development and adaptation of the computational model to the specific design context and targets play a fundamental role and thus require an accurate formalisation activity.

\section{CONCLUSION}

In this work, we presented a case study of design inspired by nature obtained by exploiting computational design strategies and additive manufacturing technologies. We emulated the structure and shape 


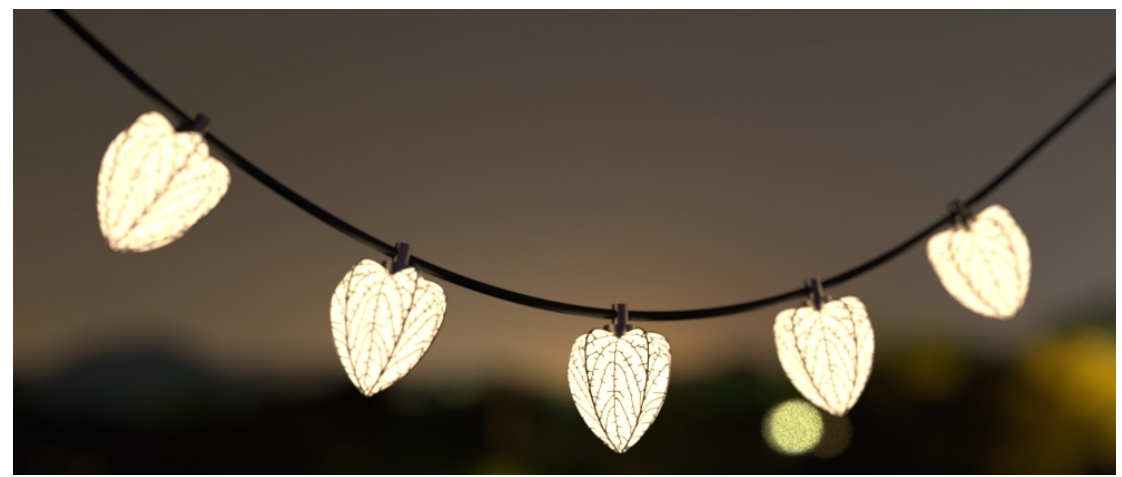

Figure 9. The final prototype in a virtual environment.

of a plant, i.e., the Physalis Alkekengi, to develop a 3D-printed lamp. We started from a mechanistic model described in the literature to formalise the $2 \mathrm{D}$ development of leaves and its venation pattern. This model has been then transformed into a Grasshopper ${ }^{\circledR}$ algorithm, to generate the parametric 3D model of our lamp, which has been prototyped using a material extrusion 3D printer.

Although in product and engineering design, Biomimicry is mostly known as a strategy aiming to solve functional problems, nature can be an inspiration also for its symbolic meaning, as done by several artists and architects. In fact, the concept of Biomimicry refers to nature both as an artist and as an engineer (Blok and Gremmen, 2016). In this case, the Alkekengi has been chosen as a model to emulate for its semantic meaning. It is known as the Chinese Lantern, so it was ideal for a lighting design project. Moreover, the way the external structure protects the internal fruit conveys a metaphor related to the concept of home comfort.

This case study has demonstrated that thanks to modern computational approaches and tools and advanced fabrication strategies, we now have more possibilities to emulate the complexity of nature in terms of its functional and aesthetic features and also of its symbolic meaning. The use of nature symbols and metaphors can contribute to developing an aesthetic of sustainable products. Biomimicry can be a means to achieve environmental targets (e.g., energy efficiency), but it could also communicate it to consumers and users, raising awareness. Moreover, the case study has allowed us to derive a design methodology for the integration of various bio-inspired aspects (functional, aesthetic, and symbolic) into the design of products. This methodology presents similar fundamental steps, regardless of the designer's objectives, features to emulate, and functions to implement. In particular, 3D printing allows the creation of complex and organic shapes. However, this does not merely require generating a 3D model of the natural form. The computational model should be designed in a specific and suitable way, allowing to maximise the advantages and, at the same time, control complexity and guarantee the feasibility of the solution.

\section{REFERENCES}

Aziz, M. S. and El sherif, A. Y. (2016), 'Biomimicry as an approach for bio-inspired structure with the aid of computation', Alexandria Engineering Journal 55(1), 707-714. https://doi.org/10.1016/j.aej.2015.10.015

Babaee, S., Pajovic, S., Rafsanjani, A., Shi, Y., Bertoldi, K. and Traverso, G. (2020), 'Bioinspired kirigami metasurfaces as assistive shoe grips', Nature Biomedical Engineering 4(8), 778-786. https://doi.org/10.1038/s41551-020-0564-3

Ball, P. (2009a), Branches: Nature's patterns: a tapestry in three parts, OUP Oxford.

Ball, P. (2009b), Shapes: nature's patterns: a tapestry in three parts, OUP Oxford.

Ball, P. (2011), Flow: Nature's patterns: A tapestry in three parts, Vol. 2, Oxford University Press.

Benyus, J. M. (1997), 'Biomimicry: Innovation inspired by nature'.

Blok, V. and Gremmen, C. (2016), 'Ecological innovation: Biomimicry as a new way of thinking and acting ecologically', Journal of Agricultural and Environmental Ethics 29, 203-217. https://doi.org/10.1007/s10806-015-9596-1

du Plessis, A. and Broeckhoven, C. (2019), 'Looking deep into nature: A review of micro-computed tomography in biomimicry', Acta Biomaterialia 85, 27-40. https://doi.org/10.1016/j.actbio.2018.12.014

du Plessis, A., Broeckhoven, C., Yadroitsava, I., Yadroitsev, I., Hands, C. H., Kunju, R. and Bhate, D. (2019), 'Beautiful and functional: A review of biomimetic design in additive manufacturing', Additive Manufacturing 27, 408-427. https://doi.org/10.1016/j.addma.2019.03.033 
El-Zeiny, R. M. A. (2012), 'Biomimicry as a problem solving methodology in interior architecture', Procedia Social and Behavioral Sciences 50, 502-512. https://doi.org/10.1016/j.sbspro.2012.08.054

Fernandes, M. C., Aizenberg, J., Weaver, J. C. and Bertoldi, K. (2020), 'Mechanically robust lattices inspired by deep-sea glass sponges', Nature Materials. https://doi.org/10.1038/s41563-020-0798-1

Fischer, S. F., Thielen, M., Loprang, R. R., Seidel, R., Fleck, C., Speck, T. and Bührig-Polaczek, A. (2010), 'Pummelos as concept generators for biomimetically inspired low weight structures with excellent damping properties', Advanced Engineering Materials 12(12), B658-B663. https://doi.org/10.1002/adem.201080065

Gokmen, S. (2013), 'A Morphogenetic approach for performative building envelope systems using leaf venetian patterns', in Stouffs, Rudi and Sariyildiz, Sevil (eds.), Computation and Performance - Proceedings of the 31 st International Conference on Education and Research in Computer Aided Architectural Design in Europe (eCAADe) - Volume 1, Faculty of Architecture, Delft University of Technology, Delft, The Netherlands, 18-20 September 2013, pp. 497-506.

Kennedy, E. B. and Marting, T. A. (2016), 'Biomimicry: Streamlining the front end of innovation for environmentally sustainable products', Research-Technology Management 59(4), 40-48. https://doi.org/10.1080/08956308.2016.1185342

Li, J., Song, C. and He, C. (2019), 'Chinese lantern in physalis is an advantageous morphological novelty and improves plant fitness', Scientific Reports 9(1), 596. https://doi.org/10.1038/s41598-018-36436-7

Nervous System (2011), 'Hyphae lamps'. Available at: https://n-e-r-v-o-u-s.com/blog/?p=1701 (accessed 24 March 2021).

Oxman, N. (2010), 'Structuring materiality: Design fabrication of heterogeneous materials', Architectural Design 80(4), 78-85. https://doi.org/10.1002/ad.1110

Prusinkiewicz, P. and Hanan, J. (1989), Lindenmayer Systems, Fractals, and Plants, Springer New York. https://doi.org/10.1007/978-1-4757-1428-9

Prusinkiewicz, P. and Lindenmayer, A. (2012), The algorithmic beauty of plants, Springer Science \& Business Media.

Prusinkiewicz, P. and Rolland-Lagan, A.-G. (2006), 'Modeling plant morphogenesis', Current Opinion in Plant Biology 9(1), 83-88. https://doi.org/10.1016/j.pbi.2005.11.015

Ren, J., Wang, Y., Yao, Y., Wang, Y., Fei, X., Qi, P., Lin, S., Kaplan, D. L., Buehler, M. J. and Ling, S. (2019), 'Biological material interfaces as inspiration for mechanical and optical material designs', Chemical Reviews 119(24), 12279-12336. https://doi.org/10.1021/acs.chemrev.9b00416

Runions, A., Fuhrer, M., Lane, B., Federl, P., Rolland-Lagan, A.-G. and Prusinkiewicz, P. (2005), Modeling and visualization of leaf venation patterns, in 'ACM SIGGRAPH 2005 Papers', SIGGRAPH '05, Association for Computing Machinery, New York, NY, USA, pp. 702-711. https://doi.org/10.1145/1186822.1073251

Salcedo, M. K., Hoffmann, J., Donoughe, S. and Mahadevan, L. (2019), 'Computational analysis of size, shape and structure of insect wings', Biology Open 8(10). https://bio.biologists.org/content/8/10/bio040774

Schaffner, M., Faber, J. A., Pianegonda, L., Rühs, P. A., Coulter, F. and Studart, A. R. (2018), '3d printing of robotic soft actuators with programmable bioinspired architectures', Nature Communications 9(1). https://doi.org/10.1038/s41467-018-03216-w

Sihite, E., Kelly, P. and Ramezani, A. (2020), 'Computational structure design of a bio-inspired armwing mechanism', IEEE Robotics and Automation Letters 5(4), 5929-5936. https://doi.org/10.1109/LRA.2020.3010217

Stevens, L., Kopnina, H., Mulder, K. and De Vries, M. (2020), 'Biomimicry design thinking education: a base-line exercise in preconceptions of biological analogies', International Journal of Technology and Design Education. https://doi.org/10.1007/s10798-020-09574-1

Tavsan, F. and Sonmez, E. (2015), 'Biomimicry in furniture design', Procedia - Social and Behavioral Sciences 197, 2285-2292. https://doi.org/10.1016/j.sbspro.2015.07.255

Volstad, N. L. and Boks, C. (2012), 'On the use of biomimicry as a useful tool for the industrial designer', Sustainable Development 20(3), 189-199. https://doi.org/10.1002/sd.1535

Wang, D., Chen, D. and Chen, Z. (2020), 'Recent progress in 3d printing of bioinspired structures', Frontiers in Materials 7, 286. https://doi.org/10.3389/fmats.2020.00286

Weerasinghe, D., Perera, S. and Dissanayake, D. (2019), 'Application of biomimicry for sustainable functionalization of textiles: review of current status and prospectus', Textile Research Journal 89(19-20), 4282-4294. https://doi.org/10.1177/0040517518821911

Yang, Y., Song, X., Li, X., Chen, Z., Zhou, C., Zhou, Q. and Chen, Y. (2018), 'Recent progress in biomimetic additive manufacturing technology: From materials to functional structures', Advanced Materials 30(36), 1706539. https://doi.org/10.1002/adma.201706539

Zhao, Y. (2010), 'Auxin biosynthesis and its role in plant development', Annual Review of Plant Biology 61(1), 49-64. https://doi.org/10.1146/annurev-arplant-042809-112308 